\title{
A GEOMETRIC PROOF OF RYLL-NARDZEWSKI'S FIXED POINT THEOREM
}

\author{
BY I. NAMIOKA AND E. ASPLUND
}

Communicated by Victor Klee, January 23, 1967

In [4], Ryll-Nardzewski gave what he called an 'old-fashioned' proof of his famous fixed point theorem. The purpose of the present note is to give an even more old-fashioned proof of the fixed point theorem. In fact, our proof uses nothing more than a category argument and the classical Krein-Milman theorem. Our terminology and notation shall be those of Kelley, Namioka et al. [2]. The following geometric lemma is essential to our proof of Ryll-Nardzewski's fixed point theorem. In case the space $E$ and the pseudo-norm $p$ in the lemma are a Banach space and its norm respectively, the lemma is an easy consequence of Lindenstrauss' work [3]. ${ }^{1}$

Lemma. Let $(E, J)$ be a locally convex Hausdorff linear topological space, let $K$ be a nonempty J-separable, weakly compact, convex subset of $E$, and let $p$ be a continuous pseudo-norm on $E$. Then for each $\epsilon>0$, there is a closed convex subset $C$ of $K$ such that $C \neq K$ and $p$-diam $(K \sim C)$ $\leqq \epsilon$, where, for any subset $X$ of $E, p-\operatorname{diam}(X)=\sup \{p(x-y): x, y \in X\}$.

Proof. Let $S=\{x: p(x) \leqq \epsilon / 4\}$; then $S$ is a weakly closed convex body. Let $D$ be the weak closure of the set of all extreme points of $K$. Since $K$ is 3 -separable, a countable number of translates of $S$ cover $K$ and hence $D$. Since $D$ is weakly compact, it is of the second category in itself with respect to the relative weak topology. Therefore there are a point $k$ of $K$ and a weakly open subset $W$ of $E$ such that $(S+k) \cap D \supset W \cap D \neq \varnothing$. Let $K_{1}$ be the closed convex hull of $D \sim W$, and let $K_{2}$ be the closed convex hull of $D \cap W$. Then, by the KreinMilman theorem and the compactness of $K_{1}$ and $K_{2}, K$ is the convex hull of $K_{1} \cup K_{2}$. Furthermore $K_{1} \neq K$. For, otherwise, by Theorem 15.2 of [2], $D \sim W$ would contain all the extreme points of $K$, contradicting the fact that $W \cap D \neq \varnothing$. Obviously $p$-diam $\left(K_{2}\right) \leqq \epsilon / 2$. Now let $r$ be a real number in $(0,1]$ and let $f_{r}$ be the map $K_{1} \times K_{2} \times[r, 1] \rightarrow K$ defined by $f_{r}\left(x_{1}, x_{2}, \lambda\right)=\lambda x_{1}+(1-\lambda) x_{2}$. Then clearly the image $C_{r}$ of $f_{r}$ is weakly closed, and it is easy to check that

1 After the draft of the present note was completed we learned that Professor J. L. Kelley knew independently that a lemma of this sort was needed for a proof of Ryll-Nardzewski's fixed point theorem. Thus he was able to give a short proof of the fixed point theorem for Banach spaces using Lindenstrauss' result. 
$C_{r}$ is convex. Moreover $C_{r} \neq K$. For, if $C_{r}=K$, then each extreme point $z$ of $K$ is of the form $z=\lambda x_{1}+(1-\lambda) x_{2}, x_{i} \in K_{i}, \lambda \in[r, 1]$. This would imply that each extreme point of $K$ is in $K_{1}$ or $K=K_{1}$, contradicting $K_{1} \neq K$. Finally, if $y \in K \sim C_{r}$, then $y$ is of the form $y=\lambda x_{1}$ $+(1-\lambda) x_{2}, x_{i} \in K_{i}, \lambda \in[0, r)$. It follows that $p\left(y-x_{2}\right)=\lambda p\left(x_{1}-x_{2}\right)$ $\leqq r d$, where $d=p$-diam $(K)<\infty$. Since $p$-diam $\left(K_{2}\right) \leqq \epsilon / 2$, we have $p$-diam $\left(K \sim C_{r}\right) \leqq \epsilon / 2+2 r d$. Therefore if we let $C=C_{r}$ for $r=\epsilon / 4 d$, the proof of the lemma is complete.

Let $Q$ be a subset of a locally convex space $E$ and let $\delta$ be a semigroup of transformations of $Q$ into $Q$. The semigroup $S$ is called noncontracting if 0 does not belong to the closure of $\{T x-T y: T \in \mathcal{S}\}$ whenever $x \neq y$ and $x, y \in Q$. Clearly $S$ is noncontracting if and only if, for $x, y \in Q$ with $x \neq y$, there is a continuous pseudo-norm $p$ (depending on $x$ and $y)$ on $E$ such that inf $\{p(T x-T y): T \in \delta\}>0$.

ThEOREM (RYLL-NARDZEWSKI). Let $Q$ be a nonempty, weakly compact, convex subset of a locally convex Hausdorff linear topological space $E$, and let $S$ be a noncontracting semigroup of weakly continuous affine maps of $Q$ into itself. Then there is a common fixed point of $s$ in $Q$.

(The following proof is not the most direct one. However it establishes an additional interesting fact concerning fixed points, also due to Ryll-Nardzewski [4]: When $S$ is finitely generated, the problem of finding a common fixed point of $\delta$ can be reduced to that of a single operator.)

Proof. By a familiar compactness argument, it is sufficient to prove that each finite subset of $\delta$ has a common fixed point in $Q$. Therefore we may assume that $\delta$ is generated by $T_{1}, T_{2}, \cdots, T_{r}$. Let $T_{0}=\left(T_{1}+T_{2}+\cdots+T_{r}\right) / r$. Then $T_{0}$ is a weakly continuous affine map of $Q$ into itself; hence there is a fixed point $x_{0}$ of $T_{0}$ in $Q$ (see, for example, Theorème 1 , Appendice of [1]). We will show that $T_{i} x_{0}=x_{0}$ for $i=1, \cdots, r$. Assume that this is not the case. Then by throwing out those $T_{i}$ 's for which $T_{i} x_{0}=x_{0}$, we may assume that $T_{i} x_{0} \neq x_{0}$ for $i=1,2, \cdots, r{ }^{2}$ Since $\delta$ is noncontracting there is a continuous pseudo-norm $p$ on $E$ and $\epsilon>0$ such that

$$
p\left(T T_{i} x_{0}-T x_{0}\right)>\epsilon \text { for all } T \text { in } \mathcal{S} \text { and } i=1, \cdots, r .
$$

Let $K$ be the closed convex hull of $\left\{T x_{0}: T \in \mathcal{S}\right\}$. Then $K$ is a weakly compact, convex, separable subset of $E$. Hence, by the lemma, there is a closed convex subset $C$ of $K$ such that $C \neq K$ and

2 Indeed if $T_{i} x_{0} \neq x_{0}$ for $i \leq m$ and $T_{i} x_{0}=x_{0}$ for $i>m$, then substitute $T_{0}{ }^{\prime}$ $=\left(T_{1}+\cdots+T_{m}\right) / m$ and the subsemigroup $S^{\prime}$ of $S$ generated by $T_{1}, \cdots, T_{m}$ for $T_{0}$ and $S$ respectively. Note that $T_{0}{ }^{\prime} x_{0}=x_{0}$. 
$p$-diam $(K \sim C) \leqq \epsilon$. Since $C \not \neq K$, there is an element $S$ in $S$ such that $S x_{0} \in K \sim C$. From $T_{0} x_{0}=x_{0}$, we see that

$$
S x_{0}=\left(S T_{1} x_{0}+S T_{2} x_{0}+\cdots+S T_{r} x_{0}\right) / r .
$$

Hence $S T_{i} x_{0} \in K \sim C$ for at least one $i$, since otherwise $S x_{0} \in C$. It follows that $p\left(S T_{i} x_{0}-S x_{0}\right) \leqq p-\operatorname{diam}(K \sim C) \leqq \epsilon$, contradicting inequality $\left(^{*}\right)$. The proof of the theorem is therefore complete.

REMARK. In the proof above $T_{0}$ could have been any convex combination $\sum_{i=1}^{r} \lambda_{i} T_{i}$ with $\lambda_{i}>0$.

\section{REFERENCES}

1. N. Bourbaki Espaces vectoriels topologiques, Chapitres I-II, Hermann Paris, 1953.

2. J. L. Kelley, I. Namioka, et al., Linear topological spaces, Van Nostrand, Princeton, N. J., 1963.

3. J. Lindenstrauss, On operators which attain their norm, Israel J. Math. 1. No. 3 (1963), 139-148.

4. C. Ryll-Nardzewski, On fixed points of semigroups of endomorphisms of linear spaces, Proc. Fifth Berkeley Symposium on Mathematical Statistics and Probability, (to appear).

UNIVERSITY OF WASHINGTON 